\title{
Molecular imaging of atheroslerotic plaque with nuclear medicine techniques (Review)
}

\author{
ORAZIO SCHILLACI, ROBERTA DANIELI, FEDERICO PADOVANO, \\ ANGELO TESTA and GIOVANNI SIMONETTI
Department of Diagnostic Imaging, Molecular Imaging, Interventional Radiology and Radiation Therapy, University Hospital 'Tor Vergata', Rome, Italy

Received January 28, 2008; Accepted March 3, 2008

\begin{abstract}
Atherosclerosis is a systemic disease of the vessel wall that mainly affects medium- and large-sized arteries, and accounts for $50 \%$ of all deaths in western countries. Imaging of atheromatous plaques has traditionally centered on assessing the degree of luminal narrowing. More recently it has become clear that it is of the utmost importance to identify the vulnerable atherosclerotic plaques responsible for the majority of life-threatening syndromes. Molecular imaging using nuclear medicine techniques such as singlephoton emission computed tomography (SPECT) and positron emission tomography (PET), has the potential to characterize the activity of atheromas. In the present review we summarize the results of radionuclide imaging in the detection of vulnerable atherosclerotic lesions.
\end{abstract}

\section{Contents}

1. Introduction: Atherosclerosis

2. SPECT imaging

3. PET imaging

4. Conclusions

\section{Introduction: Atherosclerosis}

Atherosclerosis is the most frequent cause of coronary and peripheral arterial disease, likely to become the leading cause of death worldwide by 2010 (1). It is a chronic and progressive systemic disease, with a long asymptomatic phase, characterized by the accumulation of lipids, inflammatory cells and

Correspondence to: Professor Orazio Schillaci, Viale G. Mazzini 121, I-00195 Rome, Italy

E-mail: orazio.schillaci@uniroma2.it

Key words: atherosclerosis, nuclear medicine, molecular imaging, single-photon emission computed tomography, positron emission tomography connective tissue within the intima of the arterial wall $(2,3)$. The initial pathologic abnormality is the fatty streak, due to the accumulation of serum lipoproteins and macrophages, that may develop into a mature atherosclerotic plaque, consisting of a central lipid core bounded on its lumen side by an endothelialized fibrous cap containing vascular smooth muscle cells and connective tissue.

Atherosclerosis often remains clinically silent for a long period. The symptoms, when present, are highly variable: lesion expansion is common, with a consequent limitation of blood flow, and may result in symptoms of reversible ischemia, such as angina, during periods of high metabolic demand. However, it is rupture of the plaque, with a consequent activation of circulating platelets and plasmatic proteins, and the formation of a thrombus composed by fibrin and platelets, that causes the most serious complications of atherosclerosis, such as acute coronary syndromes and stroke (1). In fact, plaque rupture is the only factor responsible for $76 \%$ of all fatal heart attacks caused by coronary thrombosis worldwide (4).

It is worth noting that several recent reports have indicated that the stability of the plaque rather than its absolute size determines the likelihood of rupture. This assumption is also based on clinical observations that acute coronary events often result from the rupture of atherosclerotic plaques at sites with no or minor luminal narrowing. The term 'vulnerable plaque' defines atherosclerotic plaques susceptible to complications such as erosion or rupture, whereas 'cardiovascular vulnerable patient' refers to a subject susceptible to an acute cardiovascular syndrome (5). Therefore, the in vivo detection of the processes underlying progressive plaque destabilization for the purpose of identifying patients in whom the disruption of a vulnerable plaque is likely to result in a clinical event, is of the utmost importance.

Several different invasive and noninvasive modalities for imaging atherosclerosis are now available, and they are clinically important for stratification and provision of early treatment. Angiography is an invasive technique that still represents the gold standard for the study of vessel lumina, but it is unable to detect atherosclerotic lesions that do not protrude into the lumen and does not give information about plaque composition (6-8). Intravascular ultrasound (IVUS), permits direct imaging of plaque and can provide a 2-dimensional cross-sectional image of the entire atheroma and vessel 
wall, with important information on the composition of individual plaques. Neverthless, IVUS is invasive, timeconsuming and expensive, and demands considerable expertise; thus its use is currently limited to only a few patients with well-defined disease, as only preselected sections of coronary and carotid vessels can be studied (9). Among noninvasive techniques, ultrasound is widely used to measure lumen stenosis due to carotid atherosclerosis, which is a cause of ischemic cerebrovascular disease, but, although easy to perform and inexpensive, it is not able to identify plaques at risk of rupture. Other noninvasive techniques, such as CT and MRI, are excellent substitutes for angiography. In particular, CT permits the evaluation of coronary calcification, an early indication of atherosclerosis (10). Moreover, contrast-enhanced multislice CT allows the high-resolution assessment of coronary artery luminal morphology, with a very high negative predictive value for exclusion of coronary disease, and it may also permit plaque characterization based on the differential attenuation of lipid, fibrous tissue, and calcium (10). MRI is capable of discriminating between lipid core, fibrous cap, intraplaque haemorrhage and calcification, and can also detect macrophage-rich lesions (2).

Most of the techniques described have been aimed at providing anatomic details of plaque size and composition. Neverthless, molecular nuclear medicine imaging has the potential to furnish functional information on cell biologic events which determine the risk of plaque rupture; moreover, besides their noninvasive nature, nuclear medicine techniques have the potential to evaluate important determinants of plaque vulnerability, taking into account specific cellular or biochemical changes that characterize these lesions. Nuclear medicine images are based on the administration of a radionuclide tracer compound to the patient, and its subsequent detection by techniques such as single-photon emission computed tomography (SPECT) and positron emission tomography (PET).

\section{SPECT imaging}

Apoptosis is an important indicator of atherosclerotic plaque vulnerability, and it occurs primarily in smooth muscle cells and macrophages. Apoptotic smooth muscle cell death leads to thinning of the fibrous cap, whereas apoptosis of macrophages results in expansion of the plaque necrotic core and positive vascular remodelling: all these features render atherosclerotic plaques prone to rupture. Therefore, the in vivo detection of apoptosis in atherosclerotic lesions can allow localization of instable plaques. Annexin A5 is a normally circulating protein which targets phosphatidylserine, a molecule expressed on the cell membrane during apoptotic cell death (2).

Technetium Tc-99m-labelled annexin A5 has thus been used for the specific targeting of vulnerable atherosclerotic lesions. In an experimental rabbit model, intense uptake in aortic lesions was observed, which was dependent on lesion severity and more pronounced in advanced atheromas, and correlated with macrophage burden, whereas no association was observed with smooth muscle cell burden (11). The impact of dietary modification and statin treatment on the incidence of apoptosis in experimental atherosclerotic lesions was later evaluated (12). A significant reduction in Tc-99m annexin A5 uptake after dietary modification and simvastatin therapy was observed; moreover, the decrease in the radiotracer uptake correlated closely with the histochemically verified reduction in apoptosis after these interventions. Given that apoptosis contributes to plaque instability, the findings of this study suggest that a reduction may be of clinical value and confirm the feasibility of Tc-99m annexin A5 for atherosclerotic plaque imaging. In a porcine model, it was demonstrated that SPECT imaging with Tc-99m annexin A5 could also be used for assessing experimental coronary atherosclerotic lesions (13).

The first clinical images with radiolabelled annexin A5 in humans were recently reported by Kietselaer et al (14), in four patients who had sustained transient ischemic attacks (TIA), before carotid endarterectomy. Tc-99 annexin A5 uptake was seen in the cervical region in the two patients with recent TIA, due to the culprit carotid vessels, whereas contralateral arteries, although similarly stenotic, did not demonstrate tracer accumulation. No uptake was also detectable in the other two patients who had suffered a TIA more than 3 months before imaging and who had been treated with antiplatelet agents and high-dose statins. After carotid endarterectomy, histological examination demonstrated that the positive Tc99m-annexin A5 uptake correlated with plaque macrophage content, whereas both patients with negative annexin imaging scans showed stable lesions with abundant smooth muscle cells and collagen, and negligible macrophage infiltration and no intraplaque haemorrhage.

High low-density lipoprotein (LDL) levels are one of the major atherogenic risk factors, which include hypertension, diabetes and smoking (1). LDL has been labelled with several radioisotopes such as Iodine-123 (I-123), Tc-99m, and Indium-111 (22). Tc-99m LDL and overall Tc-99m oxidizedLDL showed higher sensitivity for detecting atheromatous lesions (15).

Matrix metalloproteinases (MMPs) are enzymes that degrade extracellular matrix components. MMP activity has been implicated in plaque rupture, induced by the disregulation or misexpression of MMPs in the atherosclerotic lesions, and immunohistochemistry studies indicated that MMP production is predominantly in the vicinity of macrophages in human coronary atherosclerotic lesions (16). Various subtype-selective or broad-spectrum MMP inhibitors have been synthesized for treatment and assessed in clinical trials for several applications. In particular, Schäfers et al (16) studied the feasibility of SPECT imaging of MMPs in vivo using a radiolabelled broad-spectrum MMP inhibitor: they evaluated a I-123-labelled molecule (HO-CGS 27023A) in apolipoprotein E null mice which had undergone carotid artery ligation followed by high-cholesterol diet to induce rapid development of atherosclerosis. Radiotracer uptake into lesioned carotid arteries was significantly higher than normal arterial tissue from the contralateral carotid artery and carotid arteries from control mice; moreover, pre-dosing mice with unlabelled ligand prevented uptake, indicating a high level of specific binding. Microautoradiographic studies confirmed that the scintigraphically measured uptake of I-123 HO-CGS $27023 \mathrm{~A}$ in vivo was attributable to its specific accumulation in MMP-rich areas of the carotid lesion. 
Interleukin-2 (IL-2) is a cytokine which acts by binding to its receptor, mainly expressed on activated $\mathrm{T}$ lymphocytes; thus, SPECT with I-123- or Tc-99m-labelled IL-2 has been used for chronic inflammatory disease imaging (17). A group of 14 patients, with 16 carotid plaques, eligible for endarterectomy underwent Tc-99m IL-2 scan before surgery; the degree of uptake was significantly correlated with the percentage of IL-2R ${ }^{+}$cells shown by histology and the number of IL- $2 \mathrm{R}^{+}$cells assessed by flow cytometry (18). Despite the complexity of IL-2 labelling and purification, these findings suggest that $99 \mathrm{mTc}-\mathrm{IL}-2$ scintigraphy is able to detect in carotid plaques, the presence of inflammation, which plays a crucial role in promoting plaque rupture.

\section{PET imaging}

The inflammatory nature of atherosclerosis is now well recognized. From the initial phases of leukocyte recruitment, to eventual rupture of the vulnerable plaque, inflammatory mediators appear to play a central role in the pathogenesis of atherosclerosis, so that Falk defines atherosclerosis as a 'multifocal, smoldering, immuno-inflammatory disease of medium-sized and large arteries fueled by lipids' (1). Moreover, histologically vulnerable plaques are characterized by a fibrous cap $\leq 65 \mu \mathrm{m}$ thick, a lipid core occupying at least $50 \%$ of the plaque volume, numerous lipid-filled macrophages near the necrotic core and in the cap, abundant lymphocytes in the adventitia, and other inflammatory cells, such as mastcells, in the lesion. Therefore research interest has increasingly focused on inflammatory biomarkers as a means of predicting future risk of rupture.

PET images are derived from the detection of positronemitting radionuclides, labelled to biochemical and metabolic substrates. PET tracers, as a result of the higher spatial resolution of PET technology, have the potential to provide a better functional assessment of atherosclerotic plaques than SPECT tracers. In everyday clinical practice, PET is mainly used for oncology study, and fluorine-18 deoxyglucose (FDG) is the most employed radiotracer. FDG is transported into cells by glucose transporters and is phosphorylated by hexokinase enzyme to FDG-6 phosphate that is not metabolized. The degree of cellular FDG uptake is related to the cellular metabolic rate and the number of glucose transporters (19). Increased FDG uptake in tumors is due, in part, to an increased number of glucose transporters in malignant cells. A similar situation exists in inflammation: activated inflammatory cells demonstrate increased expression of glucose transporters. In addition, in inflammatory conditions, the affinity of glucose transporters for deoxyglucose is apparently increased by various cytokines and growth factors (20).

In a study aimed at investigating the relationship between the accumulation of FDG and the biologic characteristics of atherosclerotic lesions, PET imaging of vulnerable plaques was performed with a rabbit model (21). FDG accumulated to a significantly higher level in the aortas of the heritable hyperlipidemic rabbits than in those of the control ones, and uptake and the number of macrophages in the atherosclerotic lesions were strongly related. On the other hand, the correlation between FDG uptake and intimal thickness was poor. These outcomes indicate that macrophages are responsible for FDG accumulation in atherosclerotic lesions; therefore, because macrophages play a pivotal role in plaque rupture, FDG PET could be useful for the selective detection of vulnerable plaques.

Many drugs have been tested for their ability to stabilize plaque and macrophages are one of the targets of these pharmacologic therapies. Thus, the same group then used FDG PET in heritable hyperlipidemic rabbits for monitoring the therapeutic effect of probucol, an antioxidant known to reduce the extent of macrophage infiltration in atherosclerotic lesions in these animals (22). FDG uptake decreased significantly in the probucol-treated rabbits after 3 months of therapy compared with the pre-treatment period and the control group, in which it increased gradually with time. Immunohistochemical staining demonstrated that probucol treatment resulted in a reduction of macrophage infiltration in the thoracic and abdominal aortas, whereas intimal thickening was not significantly affected. These data demonstrate the capacity of FDG PET in monitoring the reduction of inflammation in plaque; moreover, it should be useful for clinically evaluating the therapeutic effect of drugs which can stabilize vulnerable plaques.

The first data on FDG PET imaging in human atherosclerotic plaque inflammation was reported by Rudd et al (23) in 8 patients who had experienced a recent carotid territory TIA and had an internal carotid artery stenosis of at least $70 \%$. They found significantly increased FDG uptake into all 8 symptomatic plaques compared to the 6 asymptomatic plaques on the contralateral side. Ex vivo analysis of endarterectomy samples demonstrated that FDG accumulated mostly in macrophages found in plaques, especially at the lipid core-fibrous cap border of the lesions. Thereafter, it was shown that FDG PET can provide in vivo a noninvasive measure of the severity of carotid plaque inflammation. In a group of 17 patients with severe carotid stenoses undergoing carotid endarterectomy, a strong correlation was found between FDG uptake and the macrophage staining from the corresponding histologic sections, whereas it did not correlate with plaque area and thickness, or area of smooth muscle cell staining (24).

The results of the above cited papers suggest that FDG PET is able to identify only those plaques that are most actively inflamed; thus not all plaques should take up significant amounts of FDG. These observations were confirmed by three studies in patients with suspected cancer evaluated by FDG PET/CT, in which a substantial disparity between CT-positive and PET-positive plaques was reported (25-27). It is well-known that CT measures calcium, an almost universal component of atherosclerosis, such that its presence or absence in the vessel wall is taken to include or exclude the presence of atherosclerosis (28); on the other hand, plaque cell biology indicates that calcification is a consequence of cell death induced by inflammation. As focal vascular wall FDG uptake was not closely associated with vascular CT calcifications, taking into account the natural history and the life cycle of atheromas, it can be hypothesized that metabolic (PET) and morphologic (CT) imaging modalities provide different types of information with respect to active or chronic lesions. Thus, FDG uptake can be a marker of 
current inflammation and potential instability, whereas CT calcification identifies past inflammation and, therefore, relative stability.

The use of FDG PET to evaluate the prevalence of inflammation in carotid atherosclerosis was recently reported (29). In a series including 100 consecutive subjects who underwent carotid artery ultrasonography for the screening of carotid atherosclerosis, inflammation was visualized by FDG imaging in about $30 \%$ of patients with documented carotid atherosclerosis. Interestingly, in a group of 216 patients submitted to FDG PET for cancer screening, increased carotid uptake was observed in the subset of patients with metabolic syndrome, suggesting a key role of this disease in vessel inflammation (30).

FDG PET imaging may also provide noninvasive longitudinal evaluation of plaque inflammation after statin therapy: 3 months of simvastatin treatment caused a reduction of FDG uptake in the atherosclerotic plaques, whereas dietary management alone did not (31). Therefore, by tracking changes in inflammation over time, serial PET could be used as a surrogate marker of anti-atheroma drug efficacy; this technique is also highly reproducible (3).

Finally, a recent study of Matter et al (32) proposed the use of fluorine-18-labelled fluorocholine for PET imaging of atherosclerosis. Increased choline accumulation was demonstrated in activated macrophages in a murine model, with a better identification of plaques in comparison with FDG.

\section{Conclusions}

Until recently, atherosclerosis imaging has focused almost entirely on defining anatomic obstructions to flow; however, a current challenge is to identify markers able to discriminate stable plaques from vulnerable ones, allowing the stratification of patients at high risk for acute cardiovascular and cerebrovascular events before clinical syndromes develop, and guidance of therapy. Molecular imaging with radionuclide tracers by SPECT and PET techniques has the potential to identify vulnerable and high-risk plaques by accurately determining their activity. In particular, apoptosis and inflammation comprise two important indicators of plaque instability that can be evaluated by nuclear medicine imaging. More work is necessary to examine whether increased radiotracer uptake correlates with a future risk of atherosclerosisrelated clinical events or plaque rupture.

\section{References}

1. Falk E: Pathogenesis of atherosclerosis. J Am Coll Cardiol 47: C7-C12, 2006.

2. Davies JR, Rudd JH and Weissberg PL: Molecular and metabolic imaging of atherosclerosis. J Nucl Med 45: 1898-1907, 2004.

3. Rudd JH, Myers KS, Bansilal S, et al: ${ }^{18}$ Fluorodeoxyglucose positron emission tomography imaging of atherosclerotic plaque inflammation is highly reproducible: implications for atherosclerosis therapy trials. J Am Coll Cardiol 50: 892-896, 2007.

4. Falk E, Shah PK and Fuster V: Atherothrombosis and thrombosis-prone plaques. In: Hurst's the Heart. Fuster V, Alexander RW, O'Rourke RA, et al (eds). 11th edition. McGraw-Hill, New York, pp1123-1139, 2004.

5. Naghavi M, Falk E, Hecht HS, et al: From vulnerable plaque to vulnerable patient - Part III: Executive summary of the Screening for Heart Attack Prevention and Education (SHAPE) Task Force report. Am J Cardiol 98: 2-15, 2006.
6. Kohler T: Imaging of carotid artery lesions: a surgeon's view. In: Syndromes of Atherosclerosis: Correlation of Clinical Imaging and Pathology. Fuster V (ed). Futura Publishing Co., Inc, Armonk, NY, pp277-289, 1996.

7. Ambrose J: Angiographic correlations of advanced coronary lesions in acute coronary syndromes. In: Syndromes of Atherosclerosis: Correlations of Clinical Imaging and Pathology. Fuster V (ed). Futura Publishing Co., Inc, Armonk, NY, pp105-122, 1996.

8. Dinsmore R: Imaging techniques in carotid and peripheral vascular disease. In: Syndromes of Atherosclerosis: Correlations of Clinical Imaging and Pathology. Fuster V (ed). Futura Publishing Co., Inc, Armonk, NY, pp277-289, 1996.

9. Schaar JA, Mastik F, Regar E, et al: Current diagnostic modalities for vulnerable plaque detection. Curr Pharm Des 13: 995-1001, 2007.

10. Botvinick EH, Perini R, Bural G, et al: The aging of the heart and blood vessels: a consideration of anatomy and physiology in the era of computed tomography, magnetic resonance imaging, and positron emission tomographic imaging methods with special consideration of atherogenesis. Semin Nucl Med 37: 120-143, 2007.

11. Kolodgie FD, Petrov A, Virmani R, et al: Targeting of apoptotic macrophages and experimental atheroma with radiolabeled annexin V: a technique with potential for noninvasive imaging of vulnerable plaque. Circulation 108: 3134-3139, 2003.

12. Hartung D, Sarai M, Petrov A, et al: Resolution of apoptosis in atherosclerotic plaque by dietary modification and statin therapy. J Nucl Med 46: 2051-2056, 2005.

13. Johnson LL, Schofield L, Donahay T, et al: $99 \mathrm{mTc}$-annexin V imaging for in vivo detection of atherosclerotic lesions in porcine coronary arteries. J Nucl Med 46: 1186-1193, 2005.

14. Kietselaer BL, Hofstra L, Dumont EA, et al: The role of labeled Annexin A5 in imaging of programmed cell death. From animal to clinical imaging. Q J Nucl Med 47: 349-361, 2003.

15. Iuliano L, Signore A, Vallabajosula S, et al: Preparation and biodistribution of $99 \mathrm{~m}$-technetium labelled oxidized LDL in man. Atherosclerosis 126: 131-141, 1996.

16. Schäfers M, Riemann B, Kopka K, et al: Scintigraphic imaging of matrix metalloproteinase activity in the arterial wall in vivo. Circulation 109: 2554-2559, 2004.

17. Signore A, Chianelli M, D'Alessandria C, et al: Receptor targeting agents for imaging inflammation/infection: where are we now? Q J Nucl Med Mol Imaging 50: 236-242, 2006.

18. Annovazzi A, D'Alessandria C, Bonanno E, et al: Synthesis of $99 \mathrm{mTc}-\mathrm{HYNIC}$-interleukin-12, a new specific radiopharmaceutical for imaging T lymphocytes. Eur J Nucl Med Mol Imaging 33: 474-482, 2006.

19. Pauwels EK, Ribeiro MJ, Stoot JH, et al: FDG accumulation and tumor biology. Nucl Med Biol 25: 317-322, 1998.

20. Yun M, Jang S, Cucchiara A, et al: ${ }^{18} \mathrm{~F}$ FDG Uptake in the large arteries: A correlation study with the atherogenic risk factors. Semin Nucl Med 32: 70-76, 2002.

21. Ogawa M, Ishino S, Mukai T, et al: ${ }^{18} \mathrm{~F}-\mathrm{FDG}$ accumulation in atherosclerotic plaques: immunohistochemical and PET imaging study. J Nucl Med 45: 1245-1250, 2004.

22. Ogawa M, Magata Y, Kato T, et al: Application of ${ }^{18} \mathrm{~F}-\mathrm{FDG}$ PET for monitoring the therapeutic effect of antiinflammatory drugs on stabilization of vulnerable atherosclerotic plaques. J Nucl Med 47: 1845-1850, 2006.

23. Rudd JH, Warburton EA, Fryer TD, et al: Imaging atherosclerotic plaque inflammation with $\left[{ }^{18} \mathrm{~F}\right]$-fluorodeoxyglucose positron emission tomography. Circulation 105: 2708-2711, 2002.

24. Tawakol A, Migrino RQ, Bashian GG, et al: In vivo ${ }^{18} \mathrm{~F}-$ fluorodeoxyglucose positron emission tomography imaging provides a noninvasive measure of carotid plaque inflammation in patients. J Am Coll Cardiol 48: 1818-1824, 2006.

25. Ben Haim S, Kupzov E, Tamir A, et al: Evaluation of ${ }^{18} \mathrm{~F}-\mathrm{FDG}$ uptake and arterial wall calcifications using ${ }^{18} \mathrm{~F}-\mathrm{FDG}$ PET/CT. J Nucl Med 45: 1816-1821, 2004.

26. Dunphy MP, Freiman A, Larson SM, et al: Association of vascular ${ }^{18} \mathrm{~F}-\mathrm{FDG}$ uptake with vascular calcification. J Nucl Med 46: 1278-1284, 2005.

27. Tatsumi M, Cohade C, Nakamoto Y, et al: Fluorodeoxyglucose uptake in the aortic wall at PET/CT: possible finding for active atherosclerosis. Radiology 229: 831-837, 2003.

28. Budoff MJ, Shavelle DM, Lamont DH, et al: Usefulness of electron beam computed tomography scanning for distinguishing ischemic from nonischemic cardiomyopathy. J Am Coll Cardiol 32: 1173-1178, 1998. 
29. Tahara N, Kai H, Nakaura H, et al: The prevalence of inflammation in carotid atherosclerosis: analysis with fluorodeoxyglucose-positron emission tomography. Eur Heart J 28: 2243-2248, 2007.

30. Tahara N, Kai H, Yamagishi S, et al: Vascular inflammation evaluated by $\left[{ }^{18} \mathrm{~F}\right]$-fluorodeoxyglucose positron emission tomography is associated with the metabolic syndrome. J Am Coll Cardiol 49: 1533-1539, 2007.
31. Tahara N, Kai H, Ishibashi M, et al: Simvastatin attenuates plaque inflammation: evaluation by fluorodeoxyglucose positron emission tomography. J Am Coll Cardiol 48: 1825$1831,2006$.

32. Matter CM, Wyss MT, Meier P, et al: ${ }^{18} \mathrm{~F}$-choline images murine atherosclerotic plaques ex vivo. Arterioscler Thromb Vasc Biol 260: 584-589, 2006. 